\title{
A indicação geográfica de vinhos finos segundo a percepção de qualidade de enófilos
}

\author{
The quality perception of Brazilian wine specialists according to geographical indications
}

\author{
Thays Ferreira Falcão ${ }^{\mathrm{I}}$ Jean Philippe Palma Révillion ${ }^{\mathrm{I}^{*}}$
}

\section{RESUMO}

As indicações geográficas (IG) representam uma forma de atributo credencial visando à distinção qualitativa do produto de uma região específica. Essa pesquisa teve como objetivo identificar as fontes de informação, o grau de valorização e de credibilidade e os fatores de qualidade relacionados ao conceito de IG em vinhos finos a partir da percepção de qualidade de enófilos de confrarias brasileiras. O estudo foi dividido em duas etapas: i) a primeira, exploratória, envolveu a revisão de literatura e consulta a especialistas para elaboração do instrumento de coleta de dados; ii) a segunda, descritiva, envolveu um pré-teste do instrumento de coleta de dados seguido pela realização de uma pesquisa via internet, com 144 enófilos. Dentre as fontes importantes para o desenvolvimento do conhecimento sobre IG, destacam-se o consumo (experiência) e a literatura especializada. $O$ estudo indica que $62 \%$ dos enófilos consultados consideram uma alta relação entre as IG e a qualidade em vinhos. Os aspectos edafoclimáticos e de produção são mais importantes na percepção de qualidade do que outros fatores relacionados ao conceito de IG.

Palavras-chave: consumidor, enófilo, indicação geográfica, qualidade em alimentos.

\section{ABSTRACT}

The geographic indication (GI) is a credential attribute, which distinguish the quality of the product of a specific region. This research aimed to identify the sources of information, the degree of appreciation and credibility, and quality factors related to the concept of GI on fine wines from the perception of wine specialists of Brazilian wine associations. The study was divided into two stages: i) the first, an exploratory one, which involved literature revision and specialists' advisement for the elaboration of the instrument for data collection; II) the second, a descriptive one, involved a previous test of the data instrument of collection followed by a survey throughout the Internet counting on the participation of 144 wine specialists. Amongst the important sources for the development of the knowledge on GI, the consumption (experience) and specialized literature are outstanding. The study indicates that $62 \%$ of the consulted wine experts identified a high relation between GI and the quality in wines. The edaphoclimatic and production aspects are more important to the perception of quality than other factors related to the GI concept.

Key words: consumer, food quality, geographic indication, wine specialist.

\section{INTRODUÇÃO}

As dimensões de qualidade consideradas em produtos alimentícios, em sua maioria, não podem ser identificadas antes da compra; por isso, os consumidores formam expectativas de qualidade que são constituídas pelas percepções individuais dos fatores intrínsecos e extrínsecos que sinalizam a potencialidade do produto em atender a essas expectativas (GRUNERT et al., 1997).

Existem também alguns atributos intrínsecos e extrínsecos que representam uma "qualidade credencial”, particularidades ou características que não podem ser comprovados pelo consumidor nem antes nem após a compra (o consumidor precisa confiar na informação transmitida na embalagem, pela mídia e por outras formas de comunicação) (DARBY \& KARNI, 1973). Para assegurar o consumidor sobre a

IPrograma de Pós-graduação em Agronegócios, Centro de Estudos e Pesquisas em Agronegócios (CEPAN), Universidade Federal do Rio Grande do Sul (UFRGS). Rua Washington Luis, 855, 90010-460, Porto Alegre, RS, Brasil. E-mail: jeanppr@gmail.com.

*Autor para correspondência. 
confiabilidade de atributos “de credencial”, são desenvolvidos certificados (SPERS et al., 1999). Porém, a percepção de qualidade de produtos “de credencial” depende de um processo de comunicação que envolve, tanto a credibilidade da fonte, quanto a habilidade de percepção do consumidor (GRUNERT et al., 1997).

Os produtos agrícolas normalmente possuem qualidades que derivam da sua região de produção. Quando essa relação é explicitada pelo estabelecimento de uma indicação geográfica (IG), sua sinalização, por meio de um selo de certificação, representa um atributo "de credencial”. No caso dos vinhos, o terroir - termo que exprime a interação entre os fatores de produção naturais e humanos - representa um fator fundamental na percepção de qualidade pelos consumidores que o reconhecem, principalmente, quando expresso em uma IG (AURIER et al., 2005; FANDOS \& FALVIÁN, 2006).

A legislação brasileira (BRASIL, 1996) estabelece que o conceito de IG envolve a definição de indicação de procedência - nome geográfico de um país, cidade região ou localidade que se tornou conhecido como centro de produção de determinado produto ou prestação de determinado serviço - e a definição de denominação de origem - nome geográfico que designa produto ou serviço cujas características ou qualidades se devam exclusiva ou essencialmente ao meio geográfico, incluídos fatores naturais e humanos.

A fim de identificar quais fatores de qualidade estão envolvidos na IG de vinhos finos sob a ótica do consumidor, este estudo buscará identificar como e quanto o consumidor enófilo (representado pelos consumidores que frequentam confrarias de vinhos) valoriza a IG em vinhos finos. Em especial, os objetivos da pesquisa contemplam: i) identificar e hierarquizar as fontes de informação dos enófilos em relação às indicações geográficas de vinhos finos; ii) avaliar o grau de importância da IG como indicador de qualidade; iii) identificar e hierarquizar os fatores relacionados a confiabilidade das IGs; e iv) identificar e hierarquizar os fatores relacionados com a percepção de qualidade de uma IG.

\section{MATERIAL E MÉTODOS}

O estudo foi dividido em duas fases: exploratória e descritiva. Na fase exploratória, foi elaborado o instrumento de coleta de dados baseado na revisão de literatura e aprimorado pelas contribuições de cinco especialistas da área: dois professores pesquisadores da Universidade Federal do Rio Grande do Sul, um pesquisador do Centro Nacional de
Pesquisas em Uva e Vinho da EMBRAPA, um pesquisador na área setorial de vinhos e um empresário participante de uma associação vitivinícola da Serra Gaúcha. Os principais objetivos dessa etapa foram identificar as dimensões de qualidade e os fatores associados à IG em vinhos finos que permitiram a estruturação da primeira versão do questionário e a construção de uma proposta conceitual para avaliação de qualidade em vinhos com IG, conforme tabela 1.

Na fase descritiva, inicialmente procedeuse ao pré-teste do instrumento, o que permitiu verificar erros de escalas e problemas de entendimento de questões, bem como reavaliar os atributos de qualidade envolvidos. A partir do pré-teste, obteve-se o instrumento final de coleta de dados, que trata de questões relacionadas à caracterização dos respondentes (sexo, idade, escolaridade e renda) e especifica a importância relativa das fontes de informação sobre IG (Tabela 2), o grau de relação entre IG e qualidade em vinhos finos, o grau de confiança das IGs estrangeiras comparadas às brasileiras, a importância relativa de fatores relacionados à confiança atribuída as IGs (Tabela 3) e, também, a importância relativa dos fatores edafoclimáticos, varietais e de produção (Tabela 4), de produto (Tabela 5) e "gerais" (Tabela 6), relacionados com a percepção de qualidade em IG. O grau de importância atribuído pelos respondentes para cada fator foi avaliado com a utilização de uma escala do tipo Likert de cinco pontos. variando entre muito baixa, baixa, média, alta e muito alta.

A aplicação do instrumento foi realizada pela internet, onde o questionário ficou disponível para acesso dos respondentes. Nessa etapa final, participaram 17 confrarias brasileiras de enófilos, ficando a amostra final constituída por 140 questionários respondidos. A seleção da populaçãoalvo para o teste e pré-teste do instrumento de coleta de dados se deu pela necessidade de atingir um consumidor com capacidade de perceber e inferir aspectos de qualidade relacionados à IG em vinhos. Dessa forma, foram convidados enófilos de confrarias, por representarem um segmento de consumidores que conhecem, de forma mais ou menos aprofundada, os aspectos relacionados à elaboração, qualidade e origem dos produtos.

Os dados foram analisados com o auxílio do software estatístico Statistical Package for the Social Sciences (SPSS), versão 13.0, e submetidos às seguintes análises estatísticas: distribuição de frequência, média aritmética e análise de variância ANOVA. 
Tabela1 - Fatores pertinentes na avaliação da qualidade de vinhos com indicação geográfica.

\begin{tabular}{|c|c|}
\hline \multirow{6}{*}{ Aspectos técnico-produtivos } & Variedade da uva utilizada ${ }^{1,2}$ \\
\hline & Adequação de técnicas de produção da uva ${ }^{1}$ \\
\hline & $\begin{array}{l}\text { Informação da safra - reconhecimento do efeito da safra no vinho }{ }^{3} \text { Adequação de } \\
\text { técnicas de produção do vinho }{ }^{1}\end{array}$ \\
\hline & Método de produção ${ }^{2}$ \\
\hline & Controle de qualidade rigoroso ${ }^{4}$ \\
\hline & Experiência no processo de produção ${ }^{4}$ \\
\hline \multirow{8}{*}{ Aspectos relacionados ao conceito de IG } & $\begin{array}{l}\text { Produto - tradicional e único }{ }^{4} \text {, produto não industrializado }{ }^{5} \text {, típico, único, autêntico e } \\
\text { natural }^{6}\end{array}$ \\
\hline & Prestígio da região de origem ${ }^{3}$ \\
\hline & Reputação do produtor ${ }^{2}$ - reflexo da cultura e história da região ${ }^{5}$ \\
\hline & Prestígio da vinícola ${ }^{3}$ \\
\hline & Reconhecimento da qualidade IG por formadores de opinião ${ }^{3}$ \\
\hline & Qualidade e confiabilidade da região de procedência ${ }^{7,8}$ \\
\hline & $\begin{array}{l}\text { Reflexo da interação entre o meio natural com o clima, o solo, o relevo e os fatores } \\
\text { humanos e de produção }{ }^{5}\end{array}$ \\
\hline & País de origem ${ }^{9}$ - nível de desenvolvimento econômico ${ }^{10}$ \\
\hline
\end{tabular}

${ }^{1}$ CHARTERS \& PETTIGREW, 2007 ; ${ }^{2}$ ORTH \& KRSKA, $2002 ;{ }^{3}$ JOVER et al., 2004 ; ${ }^{4}$ FANDOS \& FLAVIAN 2006 ; ${ }^{5}$ AURIER et al., $2005 ;{ }^{6}$ TONIETTO, $2003 ;{ }^{7}$ LOCKSHIN et al., $2006 ;{ }^{8}$ BATT \& DEAN, $2000 ;{ }^{9}$ SCHIFFMAN, $1987 ;{ }^{10}$ GUILHOTO, 2001.

\section{RESULTADOS E DISCUSSÃO}

Na análise de caracterização do público pesquisado, verificou-se que $70 \%$ dos participantes eram do sexo masculino e $60 \%$ estava na faixa etária entre 37 e 58 anos; 95\% possuía curso superior

Tabela 2 - Importância relativa das fontes para desenvolver conhecimento sobre indicação geográfica em vinhos finos.

\begin{tabular}{ll}
\hline \multicolumn{1}{c}{ Fatores } & Média \\
\hline Consumo (experiência) & $4,2^{\mathrm{a}}$ \\
Literatura especializada & $4,2^{\mathrm{a}}$ \\
Internet & $3,9^{\mathrm{b}}$ \\
Viagens & $3,8^{\mathrm{b}}$ \\
Cursos & $3,6^{\mathrm{b}}$ \\
Rótulos & $3,5^{\mathrm{bc}}$ \\
Amigos & $3,3^{\mathrm{cd}}$ \\
Informações das vinícolas & $3,1^{\mathrm{de}}$ \\
TV & $2,7^{\mathrm{e}}$ \\
\hline
\end{tabular}

* Médias com mesma letra em sobrescrito não diferem estatisticamente entre si conforme teste de comparação múltipla Dunnet T3, em nível de 0,05 de significância.

Fonte: Resultados do estudo. completo ou pós-graduação e 74\% possuía renda familiar superior a R\$3.000,00.

Dentre as fontes importantes para o desenvolvimento do conhecimento sobre IG, destacaram-se o consumo e a literatura especializada, conforme mostra a tabela 2, indicando que o consumidor pesquisado conhece e aprende sobre os vinhos de IG, considerando, principalmente, suas características intrínsecas e auxiliados por fontes menos publicitárias, como a literatura especializada. O conceito de IG representa uma dimensão de qualidade credencial que depende da confiança do consumidor na fidedignidade das informações (DARBY \& KARNI, 1973). Talvez por isso possa ser explicada a importância dada à avaliação própria dos produtos (processo sem intermediários) ou à "literatura especializada" (em princípio mais isenta).

Conforme os dados obtidos na pesquisa, $62 \%$ dos enófilos consultados consideraram uma alta ou muito alta relação entre a IG e a qualidade em vinhos. No entanto, para $64 \%$ da amostra, as IGs estrangeiras são mais ou muito mais confiáveis que as nacionais.

Conforme tabela 3, dentre os fatores relacionados à confiança associada à IG, destacaramse a credibilidade das vinícolas e credibilidade da região, evidenciando a necessidade de um trabalho sinérgico 
Tabela 3 - Importância relativa dos fatores relacionados à confiabilidade das indicações geográficas em vinhos finos.

\begin{tabular}{ll}
\hline \multicolumn{1}{c}{ Fatores } & médias \\
\hline Credibilidade das vinícolas & $4,1^{\mathrm{a}}$ \\
Credibilidade da região & $3,8^{\mathrm{ab}}$ \\
Credibilidade do país & $3,7^{\mathrm{bc}}$ \\
Credibilidade e isenção das instituições & $3,7^{\mathrm{bc}}$ \\
terceiras avaliadoras de qualidade & $3,5^{\mathrm{c}}$ \\
Credibilidade das associações & \\
\hline
\end{tabular}

* Médias com mesma letra em sobrescrito não diferem estatisticamente entre si conforme teste de comparação múltipla Dunnet T3, em nível de 0,05 de significância.

Fonte: Resultados do estudo.

entre os agentes produtivos e as instituições regionais para consolidar uma imagem de qualidade coletiva de seus produtos e de suas marcas.

De forma complementar, procurou-se identificar o grau de influência de alguns fatores para que uma região produtora de vinhos finos de qualidade tenha seu reconhecimento como IG. Para tanto, os fatores foram divididos em três grupos: i) fatores edafoclimáticos, varietais e de produção; ii) fatores relacionados ao vinho com IG; e iii) fatores "gerais" relacionados ao conceito de IG.
Conforme tabela 4, dentre os fatores edafoclimáticos, varietais e de produção, os atributos considerados como tendo mais alta relação são: o clima, o controle de qualidade da matéria-prima, o controle da produtividade, as técnicas de vinificação, as cultivares, o acompanhamento da maturação, o solo e o controle fitossanitário, sem diferenças significativas entre as médias obtidas para a importância desses atributos.

Dos fatores do produto com IG (Tabela 5), a tipicidade dos vinhos e o país de origem se destacam como fatores que possuem maior relação com a qualidade. Dentre os fatores gerais (Tabela 6), nota-se que a capacidade técnica dos enólogos está altamente relacionada com a percepção de qualidade dos vinhos com IG

Realizando uma avaliação entre as dimensões consideradas (Tabela 7), identificou-se o grupo dos fatores edafoclimáticos, varietais e de produção como o mais importante no reconhecimento de uma região produtora de vinhos de qualidade.

\section{CONCLUSÕES}

Os enófilos de vinho reconhecem a IG como um indicador de qualidade e confiabilidade influenciado em maior grau pelos aspectos edafoclimáticos e de produção envolvidos no processo produtivo e consideram de menor relevância os fatores relacionados

Tabela 4 - Importância relativa dos fatores edafoclimáticos, varietais e de produção no reconhecimento da qualidade de indicações geográficas em vinhos finos.

\begin{tabular}{|c|c|}
\hline Fatores & média \\
\hline Clima & $4,5^{\mathrm{a}}$ \\
\hline Controles analíticos de qualidade da matéria-prima (analíticos) & $4,3^{\mathrm{abc}}$ \\
\hline Controle de produtividade & $4,3^{\mathrm{abc}}$ \\
\hline Técnicas de vinificação & $4,3^{\text {abcd }}$ \\
\hline Cultivares & $4,3^{\text {abcd }}$ \\
\hline Acompanhamento da maturação & $4,2^{\text {abcd }}$ \\
\hline Solo & $4,2^{\text {abcd }}$ \\
\hline Controle fitossanitário & $4,2^{\text {abcd }}$ \\
\hline Sanidade das mudas & 4,2 bcde \\
\hline Envelhecimento dos vinhos & $4,1^{\text {bcde }}$ \\
\hline Corte dos vinhos & 4,0 bcde \\
\hline Práticas culturais (desbrote, raleio de cachos, desfolha, etc.) & 4,0 bcdef \\
\hline Equipamentos e atividades relacionadas com o desengace, a moagem e a prensagem das uvas & $4,0^{\text {cdef }}$ \\
\hline Sistema de colheita e transporte & $4,0^{\text {def }}$ \\
\hline Sistema de condução & $3,9^{\text {efg }}$ \\
\hline Idade média do vinhedo & $3,8^{\mathrm{fg}}$ \\
\hline Sistema de engarrafamento & $3,6^{\mathrm{g}}$ \\
\hline
\end{tabular}

* Médias com mesma letra em sobrescrito não diferem estatisticamente entre si conforme teste de comparação múltipla Dunnet T3, em nível de 0,05 de significância.

Fonte: Resultados do estudo.

Ciência Rural, v.40, n.2, fev, 2010. 
Tabela 5 - Importância relativa dos fatores relacionados ao produto (vinho fino) com indicação geográfica.

\begin{tabular}{lc}
\hline Fatores & média \\
\hline Tipicidade & $4,0^{\mathrm{a}}$ \\
País de origem & $3,9^{\mathrm{ab}}$ \\
Cultura e história & $3,7^{\mathrm{bc}}$ \\
Originalidade & $3,5^{\mathrm{c}}$ \\
Menor interferência tecnológica & $3,0^{\mathrm{d}}$ \\
\hline
\end{tabular}

* Médias com mesma letra em sobrescrito não diferem estatisticamente entre si conforme teste de comparação múltipla Dunnet T3, em nível de 0,05 de significância.

Fonte: Resultados do estudo.

ao conceito de IG (tanto vinculados ao produto, quanto vinculados ao conceito geral). No entanto, esse importante segmento de consumidores confia mais nos vinhos finos com IGs estrangeiras do que as nacionais, o que ratifica a necessidade do desenvolvimento de esforços constantes para incrementar a credibilidade das vinícolas e de todas as regiões produtoras brasileiras junto aos consumidores.

É possível perceber, também, que, para esses formadores de opinião, a confiança associada às IGs está fortemente relacionada à credibilidade das vinícolas e da região, diferentemente do que se observa em alguns países europeus, em que a região de procedência, por si só, representa uma forte referência de confiança, qualidade e tradição.

Dentre as limitações da pesquisa, é importante enfatizar que os resultados representam as percepções de um grupo de consumidores que detém um conhecimento mais amplo e aprofundado sobre os direcionadores de qualidade em vinhos finos do que o consumidor médio, o que não permite extrapolar os resultados deste estudo para caracterizar o comportamento dos consumidores brasileiros em geral.

Tabela 6 - Importância relativa de fatores “gerais” relacionados ao conceito de indicação geográfica em vinhos finos.

\begin{tabular}{lc}
\hline \multicolumn{1}{c}{ Fatores } & média \\
\hline Capacidade técnica enólogos & $4,3^{\mathrm{a}}$ \\
Capacidade técnica viticultores & $4,3^{\mathrm{a}}$ \\
Tradição na elaboração & $4,0^{\mathrm{b}}$ \\
Tradição da vinícola & $4,0^{\mathrm{b}}$ \\
Tradição dos vitivinicultores & $4,0^{\mathrm{b}}$ \\
Locais de comercialização & $3,2^{\mathrm{c}}$ \\
Beleza natural da região & $2,6^{\mathrm{d}}$
\end{tabular}

*Médias com mesma letra em sobrescrito não diferem estatisticamente entre si conforme teste de comparação múltipla Dunnet T3, em nível de 0,05 de significância.

Fonte: Resultados do estudo.
Tabela 7 - Importância relativa dos grupos de fatores edafoclimáticos, varietais e de produção, gerais e do vinho com indicação geográfica.

\begin{tabular}{lc}
\hline \multicolumn{1}{c}{ Fatores } & Média \\
\hline Fatores edafoclimáticos, varietais e de produção & $4,1^{\mathrm{a}}$ \\
Fatores gerais & $3,8^{\mathrm{b}}$ \\
Fatores do vinho com IG & $3,6^{\mathrm{b}}$ \\
\hline
\end{tabular}

* Médias com mesma letra em sobrescrito não diferem estatisticamente entre si conforme teste de comparação múltipla Dunnet T3, em nível de 0,05 de significância.

Fonte: Resultados do estudo.

\section{AGRADECIMENTOS}

Os autores agradecem aos especialistas e a todos os confrades de vinho e confrarias que se dispuseram a participar da pesquisa, em especial à Sociedade Brasileira de Amigos do Vinho - Rio Grande do Sul. Este estudo contou com bolsa da Coordenação de Aperfeiçoamento de Pessoal de Nível Superior - CAPES.

\section{REFERÊNCIAS}

AURIER, P. et al. Exploring terroir product meaning for the consumer. Anthropology of Food, n.4, 2005. Disponível em: <http://aof.revues.org>. On line. Acesso em: 27 ago. 2009.

BATT, P.J.; DEAN, A. Factors influencing the consumer's decision. Australian and New Zealand Wine Industry Journal, v.15, n.4, p.34-41, 2000.

BRASIL, Lei n.9279 de 14/05/1996. Regula direitos e obrigações relativos à propriedade industrial. Planalto. Disponível em: <http://planalto.gov.br/ccivil/LEIS/L9279.htm>. On line. Acesso em: 25 fev. 2006.

CHARTERS, S.; PETTIGREW, S. The dimensions of wine quality. Food Quality and Preference, v.18, n.7, p.9971007, 2007. Disponível em: <http://www.sciencedirect.com>. Acesso em: 20 out. 2006 doi: 10.1016/j.foodqual.2007.04.003.

DARBY, M.R.; KARNI, E. Free competition and the optimal amount of fraud. Journal of Law and Economics, v.16, n.1, p.67-88, 1973. Disponível em: <http://www.journals.uchicago.edu>. Acesso em: 14 jul. 2006 doi: 10.1086/466756.

FANDOS, C.; FLAVIÁN, C. Intrinsic and extrinsic quality attributes, loyalty and buying intention: an analysis for PDO product. British Food Journal, v.108, n.8, p.646-662, 2006. Disponível em: <http://www.emeraldinsight.com>. Acesso em: 23 nov. 2007. doi: 10.1108/00070700610682337.

GRUNERT, K.G. et al. Innovation in the food sector: a revised framework. In: TRAIL,B.; GRUNERT, K.G. Product and process innovation in the food industry. Suffolk: Chapman \& Hall, 1997. Cap.15, p.213-226.

GUILHOTO, L.F.M. A influência do país de origem na percepção do consumidor sobre a qualidade dos produtos. Caderno de Pesquisas em Administração, v.8, n.4, p.53-68, 2001. 
Disponível em: <http://www.ead.fea.usp.br>. Acesso em: 27 ago. 2009.

JOVER, A.J. et al. Measuring perceptions of quality in food products: the case of red wine. Food Quality and Preference, v.15, n.5, p.453-446, 2004. Disponível em: <http:// sciencedirect.com>. Acesso em: 02 jan. 2007. doi: 10.1016/ j.foodqual.2003.08.002.

LOCKSHIN, L. et al. Using simulations from discrete choice experiments to measure consumer sensitivity to brand, region, price, and awards in wine choice. Food Quality and Preference, v.17, n.3-4, p.166-178, 2006. Disponível em: <http://www.sciencedirect.com>. Acesso em: 14 set. 2007. doi: 10.1016/j.foodqual.2005.03.009.

ORTH, U.R.; KRSKA, P. Quality signals in wine marketing: the role of exhibition award. International Food and
Agribusiness Management Review, n.4, p.385-397, 2002. Disponível em: <http://ageconsearch.umn.edu>. Acesso em: 27 ago. 2009.

SCHIFFMAN, L.G. Consumer behavior. Englewood Cliffs: Prentice Hall, 1987. 725p.

SPERS, E.E. et al. Dungullin state:certificação de qualidade na agricultura australiana. In: SEMINÁRIO INTERNACIONAL PENSA DE AGRIBUS INESS, 9., 1999, São Paulo, Brasil. Anais... São Paulo: Programa de Estudos e dos Negócios do Sistema Agroindustrial, 1999. Disponível em: <http:// www.fundacaofia.com.br>. Acesso em: 31 ago. 2009.

TONIETTO, J. Uvas viníferas para processamento. Sistema de Produção, n.4, 2003. Versão Eletrônica. Disponível em: <http://sistemasdeproducao.cnptia.embrapa.br>. On line. Acesso em: 31 ago. 2009. 